\title{
Revista da Faculdade de
}

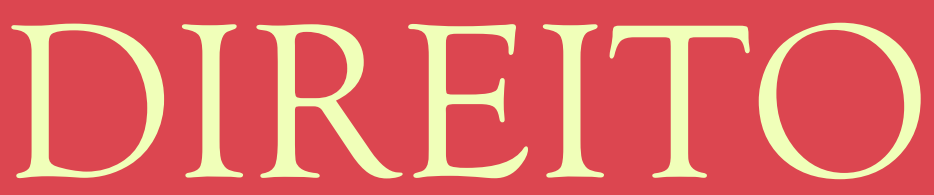

$\begin{array}{llll}\mathrm{U} & \mathrm{F} & \mathrm{P} & \mathrm{R}\end{array}$

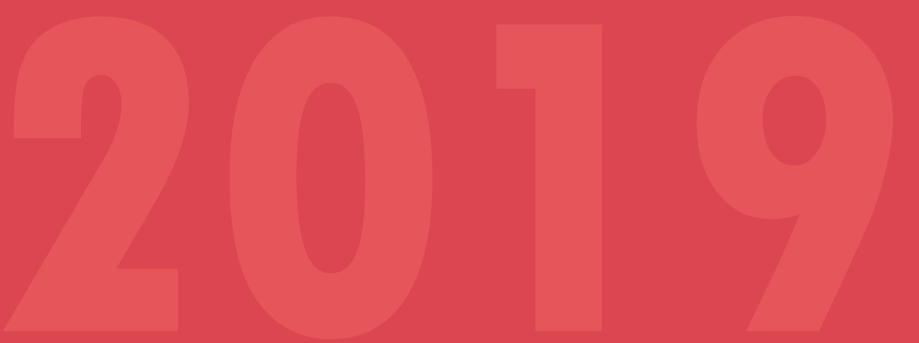



2019 • Vol. 64 • Número 1 • ISSN 2236-7284

Revista da Faculdade de

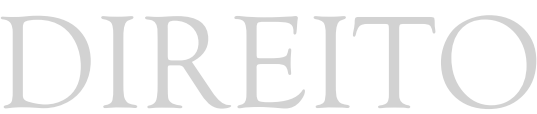

U F $\quad$ P R 


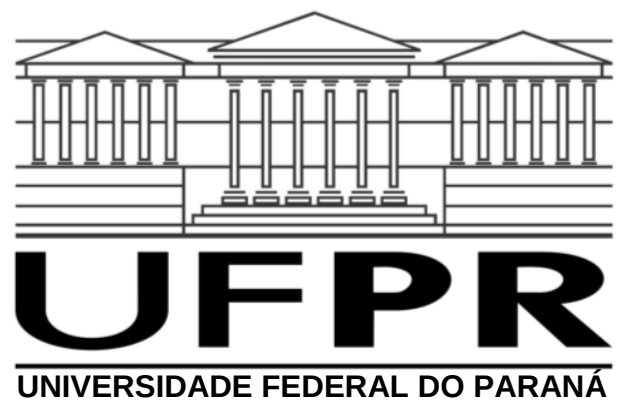

Reitor - Prof. Dr. Ricardo Marcelo Fonseca

Vice-Reitora - Prof. ${ }^{a}$ Dr. ${ }^{a}$ Graciela Inês Bolzón de Muniz

Pró-Reitor de Pesquisa e Pós-Graduação - Prof. Dr. Francisco de Assis Mendonça

SETOR DE CIÊNCIAS JURÍDICAS

Diretora - Prof. ${ }^{a}$ Dr. ${ }^{a}$ Vera Karam de Chueiri

Vice-Diretora - Prof. ${ }^{a}$ Dr. ${ }^{a}$ Maria Cândida Pires Vieira do Amaral Kroetz

Coordenador do Programa de Pós-Graduação em Direito - Prof. Dr. Fabricio Ricardo de Limas Tomio

Vice-Coordenador do Programa de Pós-Graduação em Direito - Prof. Dr. Sérgio Said Staut Júnior

FACULDADE DE DIREITO

Chefe do Departamento de Direito Privado - Prof. ${ }^{a}$ Dr. ${ }^{a}$ Katya Kozicki

Chefe do Departamento de Direito Público - Prof. Dr. Rodrigo Luís Kanayama

Chefe do Departamento de Direito Civil e Processual Civil - Prof. Dr. Clayton de Albuquerque Maranhão Chefe do Departamento de Direito Penal e Processual Penal - Prof. Dr. João Gualberto Garcez Ramos 


\section{Revista da Faculdade de}
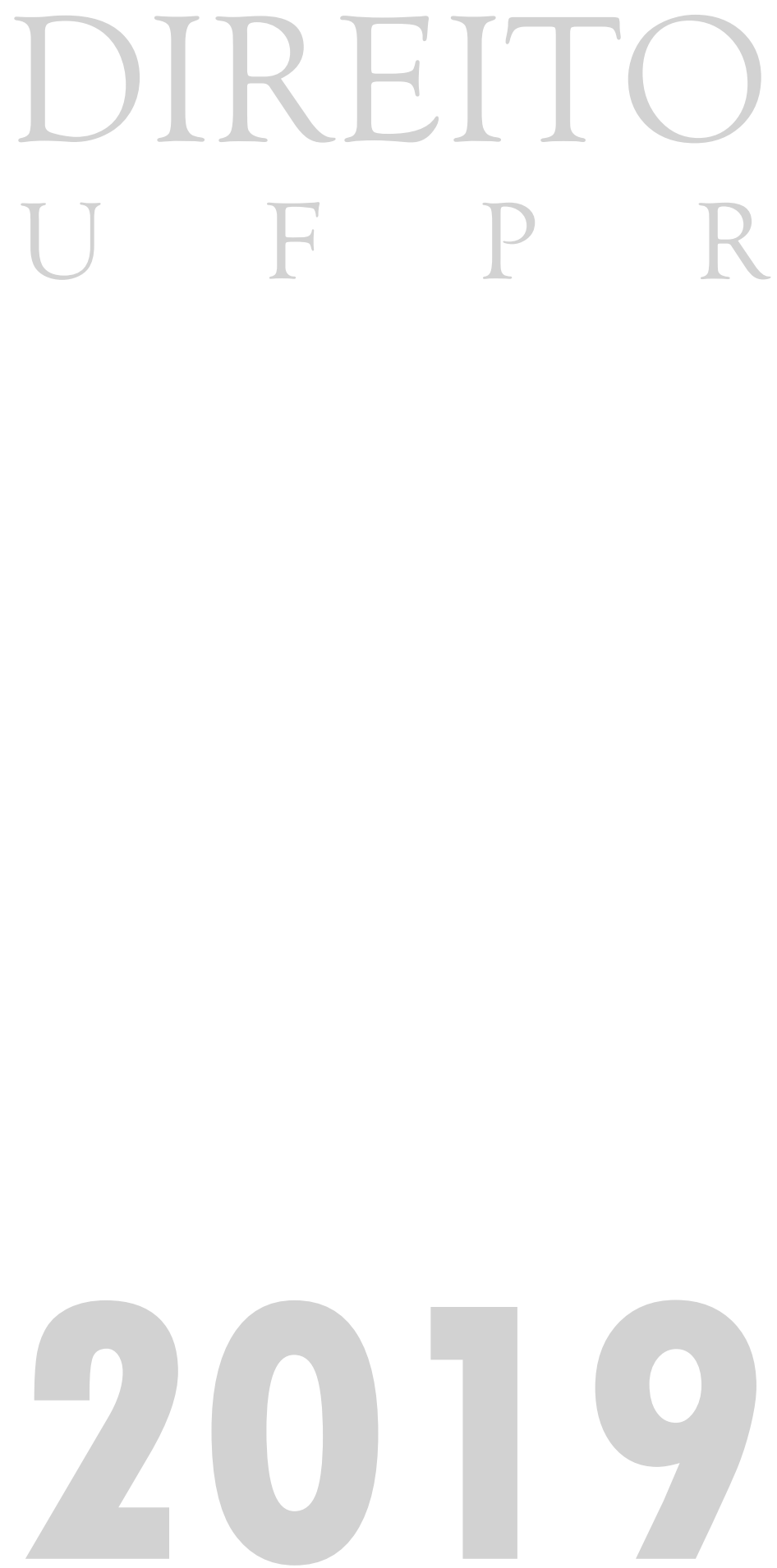


\section{Revista da Faculdade de Direito UFPR}

\section{Objetivo}

A Revista da Faculdade de Direito UFPR é uma publicação quadrimestral de trabalhos científicos inéditos da área jurídica, nacional ou estrangeira. A Revista tem interesse na publicação de artigos, análises de decisões judiciais, comentários críticos jurisprudenciais e resenhas críticas. A linha editoria da Revista prestigia o movimento de aproximação do direito com outras áreas do conhecimento e o compromisso da universidade pública com a construção de uma sociedade democrática, justa e solidária.

\section{Editora-chefe}

Prof. ${ }^{a}$ Dr. ${ }^{a}$ Estefânia Maria de Queiroz Barboza

Editor adjunto

Prof. Dr. Ilton Norberto Robl Filho

Editores executivos

Gustavo Dalpupo de Lara

Maria Teresa Vasconcelos Guimarães da Costa

Rennan Klingelfus Gardoni

Revisor de texto

Márcio Eduardo Zuba

\section{Conselho Editorial}

Antonio Carlos Wolkmer (Universidade Federal de Santa Catarina), António José Avelãs Nunes (Universidade de Coimbra), Antonio Manuel Hespanha (Universidade Nova de Lisboa), Bethania Assy (PUC-Rio de Janeiro e Universidade Estadual do Rio de Janeiro), Claudia Lima Marques (Universidade Federal do Rio Grande do Sul), Claudia Perroné-Moises (Universidade de São Paulo), David Ritchie (Mercer University School of Law, Georgia, EUA), Giovanni Cazzetta (Università degli Studi di Ferrara), José Antônio Peres Gediel (Universidade Federal do Paraná), José Carlos Moreira da Silva Filho (Universidade do Vale do Rio dos Sinos), Juliana Neuenschwander Magalhães (Universidade Federal do Rio Janeiro), Katya Kozicki (Universidade Federal do Paraná), Laymert Garcia dos Santos (Universidade Estadual de Campinas), Marcelo Cattoni (Universidade Federal de Minas Gerais), Menelick de Carvalho Netto (Universidade de Brasília), Paulo Luiz Netto Lôbo (Universidade Federal de Alagoas), Ricardo Marcelo Fonseca (Universidade Federal do Paraná), Roberto Gargarella (Universidad de Buenos Aires), Samuel Barbosa (Universidade de São Paulo).

Pareceristas permanentes: Alexandre Bernardino Costa (Universidade de Brasília), Cristiano Paixão (Universidade de Brasilia), Eduardo Henrique Lopes Figueiredo (USM - Universidade do Sul de Minas), Fabiola Albuquerque (Universidade Federal de Pernambuco), Jeanine Nicolazzi Phillippi (Universidade Federal de Santa Catarina), José Ramón Narváez Hernández (UNAM - México), Osvaldo Lopes Ruiz (Universidade de Mendoza).

Os membros do Conselho Editorial, o corpo docente do Programa de Pós-Graduação em Direito da UFPR, pesquisadores da Faculdade de Direito, além dos professores e pesquisadores de outras instituições nomeados ad-hoc também serão responsáveis pelos pareceres dos artigos submetidos para publicação.

Endereço para contato:

Faculdade de Direito da UFPR - PPGD

Praça Santos Andrade, 50 - Centro - $3^{\circ}$ andar - CEP 80020-300 - Curitiba, PR, Brasil Telefone/Phone +55 (41) 3310-2685 E-mail: revistadireito@ufpr.br

R454 DADOS INTERNACIONAIS DE CATALOGAÇÃO NA PUBLICAÇÃO
Revista da Faculdade de Direito UFPR / Universidade Federal do
Paraná, Programa de Pós-Graduação em Direito.- ano 1, n. 1
(dez. 1953)- - - Curitiba: UFPR, 1953-
v.; $24 \mathrm{~cm}$.
Quadrimestral.
Endereço eletrônico: http://ojs.c3sl.ufpr.br/ojs/index.php/direito

ISSN 2236-7284.

1. Direito. I. Universidade Federal do Paraná. Programa de Pós-Graduação em Direito.

Catalogação na Fonte UFPR - Sistema de Bibliotecas - SiBi

Intercâmbio UFPR

SIBI/Biblioteca Central / Secão de Intercâmbio

Rua General Carneiro, 370 - Centro - CEP 80020-300 - Curitiba, PR, Brasil

E-mail: bc@ufpr.br - Fone / Fax: (41) 3360-5280

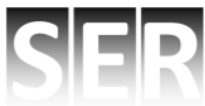

Pró-Reitoria de Pesquisa e Pós-Graduação

Programa de Apoio à Publicação de Periódicos da UFPR

Sistema Eletrônico de Revistas - SER

www.prppg.ufpr.br 


\section{CONTENTS}

\section{ARTIGOS}

9 O desenvolvimento das normas jus cogens em relação ao reconhecimento do direito à propriedade coletiva dos povos indígenas à luz da sentença do caso Povo Indígena Xucuru e seus membros versus Brasil (DOI 10.5380/rfdufpr.v64i1.61647)

The development of the jus cogens norms and the recognition of the right to the property of the indigenous peoples in the light of the case of the Indigenous People Xucuru and its members versus Brazil

Waldir De Jesus Brabo Ferreira Júnior

Natália Mascarenhas Simões Bentes

39 Representação dos trabalhadores terceirizados em face da ampliação da permissão jurídica da terceirização no Brasil: análise na perspectiva do trabalho decente e de seu imperativo de proteção dos direitos humanos (DOI 10.5380/rfdufpr.v64i1.61985)

Representation of outsourced workers in face of the enlargement of the legal permission of outsourcing in Brazil: An analysis by the perspective of decent work and its imperative for the protection of human rights

\section{Silvio Beltramelli Neto}

\section{Laís Silveira}

63 Criminologia midiática e a campanha "Crack, nem pensar": refletindo sobre a falta de pensamento (DOI 10.5380/rfdufpr.v64i1.62382)

Media criminology and the campaign “Crack, nem pensar”: Reflecting on a lack of thought

Guilherme Michelotto Böes

Augusto Jobim Do Amaral

97 Para uma concepção epistemológica da incerteza (DOI 10.5380/rfdufpr.v64i1.62405)

For an epistemological conception of uncertainty

Celso Luiz Ludwig

119 A telemática como instrumento de labor: teletrabalho e hiperconexão (DOI 10.5380/rfdufpr.v64i1.63698)

Telematic as a labour instrument: Teleworking and hyperconnection

Denise Pires Fincato

Julise Carolina Lemonje 
137 A judicialização do refúgio no STJ: deferência ao Executivo e incoerência interpretativa (DOI 10.5380/rfdufpr.v64i1.64908)

The judicialization of refuge in the STJ: Deference to the Executive branch and interpretative incoherence

Breno Baía Magalhães

Gabriella Thaís Sousa Corrêa

165 Judicialização das práticas trabalhistas: a questão da pejotização na jurisprudência do TRT-1 (DOI 10.5380/rfdufpr.v64i1.64979)

Judicialization of labor practices: The question of pejotization (independent-contractor-only hiring policy) in the TRT-1 jurisprudence

Felipe Dutra Asensi

Isabela Pfister Gonçalves

187 A incidência das contribuições previdenciárias sobre a produção rural e seus aspectos controvertidos (DOI 10.5380/rfdufpr.v64i1.65407)

The social security contributions incidence on rural production and its controversial aspects

André Mendes Moreira

Luiz Felipe Mendes Álvares da Silva Campos 